\title{
Feasibility of an eHealth application "OncoKompas" to improve personalized survivorship cancer care
}

\author{
S. Duman-Lubberding ${ }^{1,6,7}$ • C.F. van Uden-Kraan ${ }^{1,2,6,7}$ • F. Jansen ${ }^{1,6,7}$ • B.I. Witte ${ }^{3}$ • \\ L.A. van der Velden ${ }^{4}$ - M. Lacko $^{5}$ • P. Cuijpers ${ }^{2,6}$ • C.R. Leemans ${ }^{1,7}$ • I.M. Verdonck-de Leeuw ${ }^{1,2,6,7}$
}

Received: 28 April 2015 / Accepted: 27 October 2015 /Published online: 12 November 2015

(C) The Author(s) 2015. This article is published with open access at Springerlink.com

\begin{abstract}
Purpose The purpose of this study was to investigate the feasibility of an online self-management application (OncoKompas) among cancer survivors. In OncoKompas, cancer survivors can monitor their quality of life (QOL) via participant reported outcomes (PROs) ("Measure"), which is followed by automatically generated individually tailored feedback ("Learn") and personalized advice on supportive care services ("Act").

Methods A pretest-posttest design was used, conducting a survey before providing access to OncoKompas, and 2 weeks after, followed by an interview by a nurse. Adoption was defined as the percentage of cancer survivors that agreed to participate in the study and returned the T0 questionnaire. Implementation was defined as the percentage of participants that actually used OncoKompas as intended (T1). General satisfaction was assessed based on the mean score of three
\end{abstract}

I.M. Verdonck-de Leeuw

IM.Verdonck@vumc.nl

1 Otolaryngology/Head and Neck Surgery, VU University Medical Center, Amsterdam, The Netherlands

2 Clinical Psychology, VU University, Amsterdam, The Netherlands

3 Epidemiology and Biostatistics, VU University Medical Center, Amsterdam, The Netherlands

4 Otolaryngology/Head and Neck Surgery, Leiden University Medical Center, Leiden, The Netherlands

5 Otolaryngology/Head and Neck Surgery, Maastricht University Medical Center, Maastricht, The Netherlands

6 EMGO Institute for Health and Care Research, VU University and VU University Medical Center, Amsterdam, The Netherlands

7 Cancer Center Amsterdam, VU University Medical Center, Amsterdam, The Netherlands study-specific questions: (1) general impression of OncoKompas, (2) the user-friendliness, and (3) the ability to use OncoKompas without assistance (10-point Likert scales). Furthermore, satisfaction was measured with the Net Promotor Scale (NPS).

Results OncoKompas was feasible with an adoption grade of $64 \%$, an implementation grade of $75-91 \%$, a mean satisfaction score of 7.3, and a positive NPS (1.9). Sociodemographic and clinical factors and QOL were not associated with satisfaction. Several facilitators and barriers related to the feasibility of OncoKompas were identified.

Conclusion OncoKompas is considered feasible, but has to be further improved. In order to enhance feasibility and increase satisfaction, we have to balance the time it takes to use OncoKompas, measurement precision, and tailoring towards personalized advices.

Keywords Neoplasms · Self-management $\cdot$ EHealth · Supportive cancer care $\cdot$ Quality of life $\cdot$ Lifestyle

\section{Introduction}

Cancer and cancer treatment have a large impact on quality of life (QOL). Head and neck cancer (HNC) has a specific impact on survivors. In addition to symptoms such as fatigue, $\mathrm{HNC}$ survivors are confronted with oral dysfunction, speech and swallowing problems, and related social withdrawal and psychological distress. All of these symptoms can deteriorate quality of life [1,2] and increase survivors' needs for supportive care services. Several recent papers report on the need for improving survivorship cancer care [3, 4] to enhance quality of life and diminish societal discrimination.

It is essential that cancer survivors have access to optimal supportive care services including self-management options. 
Access to supportive care may be hampered by current changes in the health care system, e.g., limited time of health care providers and centralization of care [5]. To improve accessibility, cancer survivors are expected to adopt an active role in managing their own care. Self-management is defined by McCorkle et al. [6] as "those tasks that individuals undertake to deal with the medical, role, and emotional management of their health condition(s)." Alongside usual care, selfmanagement options can be (cost-)effective and improve quality of life [3]. Although there is evidence that supportive cancer care can be effective, referral rates are low and many survivors have unmet needs. Innovating supportive cancer care includes incorporation of self-management and eHealth, implementation of evidence-based approaches to monitor QOL $[7,8]$, and redesign of the organization of supportive care according to participant centered models of care (e.g., the chronic care model, disease management, and stepped care) $[3,7,9]$.

We developed an eHealth application "OncoKompas" with the aim to facilitate and innovate the access to supportive cancer care. In OncoKompas, cancer survivors can monitor their QOL by means of participant reported outcomes (PROs) ("Measure"), which is followed by automatically generated tailored feedback ("Learn") and personalized advice on supportive care services (“Act”). To ensure sustainable implementation of OncoKompas, we followed participatory design principles. Cancer survivors and health care professionals (HCPs) were involved in each step of the development process [10-12] (Fig. 1). A qualitative assessment of needs among cancer survivors and HCPs (step 1) showed that cancer survivors are interested in an eHealth application that targets personalized access to supportive care and that HCPs expect that an eHealth application could optimize survivorship care

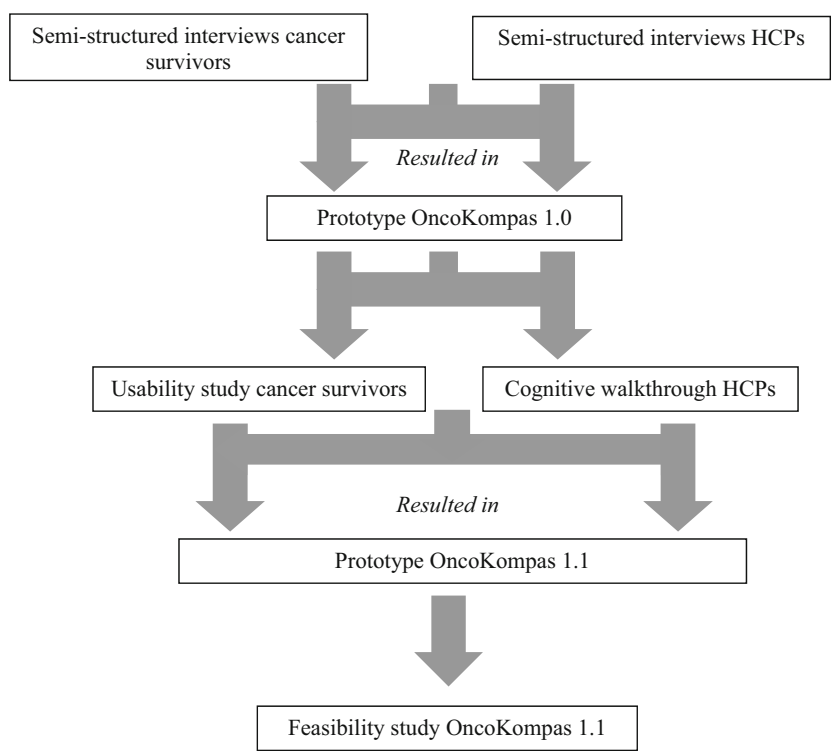

Fig. 1 Flow chart development process OncoKompas based on participatory design principles
[13]. A prototype of the eHealth application, "OncoKompas", was developed. Existing applications were used as examples to build the application $[14,15]$. OncoKompas was developed together with mixed teams consisting of cancer survivors and medical specialists as well as allied health professionals. In step 2, the usability of a prototype was tested among both cancer survivors and HCPs, targeting system quality (ease of use), content quality (usefulness and relevance), and service quality (the process of care provided) [16, 17]. HCPs raised several points for improvement regarding the ease of use and usefulness of the application, resulting in improved persuasiveness and improved tailoring. Usability tests among cancer survivors identified some weaknesses in the user interface that resulted in adjustments, e.g., clearer user instructions [18]. Based on these findings, the prototype of OncoKompas was optimized. The next step (step 3) in the developmental cycle of OncoKompas is to investigate the feasibility in clinical practice.

The purpose of the present study was to investigate the feasibility of OncoKompas: adoption (intent to use OncoKompas), usage (actual use of OncoKompas), and satisfaction with OncoKompas among cancer survivors. Secondary aims were to investigate which sociodemographic and clinical factors are associated with the feasibility of OncoKompas and to obtain insight in possible barriers and facilitators of the feasibility of OncoKompas.

This feasibility study will provide insight into factors that contribute to the development and usage of eHealth applications among head and neck cancer survivors.

\section{Methods}

A pretest-posttest design was used, conducting a survey before providing cancer survivors access to OncoKompas (T0) and 2 weeks after (T1). After participants completed the T1 survey, they were interviewed by a nurse specialized in oncology to obtain more in-depth insight into the feasibility of OncoKompas.

\section{Study sample and procedures}

Between January and July 2013, all eligible cancer survivors from the Departments of Otolaryngology-Head and Neck Surgery from VU University Medical Center, Amsterdam, University Medical Center Leiden, and University Medical Center Maastricht, The Netherlands were invited by an oncology nurse or head and neck surgeon. Participants were eligible if they (1) were treated for head and neck cancer with curative intent with a maximum of 2 years prior, (2) were 18 years or older, (3) were able to write, read, and speak Dutch, (4) had some Internet experience, and (5) had access to the Internet at home. 
If participants agreed to participate, they were asked to complete and return a paper-and-pencil questionnaire (T0). Subsequently, participants were contacted to provide them with a login code for OncoKompas at home. An interview was scheduled with an oncology nurse from their hospital 2 weeks later. Prior to their appointment with the nurse, cancer survivors were asked to fill in the post-test questionnaire (T1). During the consultation with the nurse, attention was paid to perceived usefulness of the tailored advice and personalized referral to supportive care services, as provided by OncoKompas. Oncology nurses made standardized interview reports. The scheme consisted of two main components: part A for survivors who completed OncoKompas and part B for survivors who did not complete Oncokompas. Key questions included in part A comprise the following: (1) "How would the survivor describe their experience with OncoKompas?", (2) "Did the survivor view their personalized advice and supportive care options? If not, why?", and (3) "Did the survivor find the results applicable to their personal situation?". Key questions included in part B comprise the following: (1) Why did the survivor not complete OncoKompas?", (2) "What should be changed in the application to enable the survivor to complete OncoKompas?", and (3) "What aspects did the survivor miss in OncoKompas that prevented the survivor to complete the application?". Nurses completed the scheme during the consultation with the survivors and supplemented these following the consultation.

Technical support was offered by two researchers (SL and FJ) when problems occurred with the access or use of OncoKompas. The researchers recorded an entry in a logbook with each technical problem.

The study was conducted according to regular procedures of the local ethical committee of the VU University Medical Center, Amsterdam. All participants signed informed consent.

\section{Intervention "OncoKompas"}

OncoKompas can be considered as both a screening and a monitoring tool and consists of three components: (1) measure, (2) learn, and (3) act. In the "Measure" component, cancer survivors can independently complete PROs targeting the following QOL domains: physical functioning, psychological functioning, social functioning, healthy lifestyle, and existential issues. Besides these domains for cancer survivors in general, a specific domain, containing topics for head and neck cancer patients, is allocated (Table 1). Specific PROs were selected by the project team in collaboration with teams of experts, based on Dutch practice guidelines and literature searches. Data from the "Measure" component are processed in real-time and linked to tailored feedback to the cancer survivor in the "Learn" component. All algorithm calculations are based on available cut-off scores, or they are defined based on Dutch practice guidelines, literature searches, and/or consensus by teams of experts. In the "Learn" component, a compass metaphor is used to summarize overall well-being. Following this, feedback is provided to the participant on the level of the topics (e.g., depression and fatigue) by means of a three-color system: green (no elevated well-being risks), orange (elevated well-being risks), and red (seriously elevated well-being risks) (first-degree algorithms). Cancer survivors receive elaborated personalized information on the outcomes, e.g., on the topic depression, information is provided on the symptoms of depression and the proportion of cancer survivors who suffer from depressive symptoms.

Special attention is paid to clusters of interrelated symptoms. For example, feedback on the association between depression and fatigue is provided, if a participant has an orange or a red score on depression as well as on fatigue. The

Table 1 Overview of topics OncoKompas

\begin{tabular}{|c|c|c|c|c|c|}
\hline $\begin{array}{l}\text { Psychological quality } \\
\text { of life }\end{array}$ & Physical quality of life & Social quality of life & Healthy lifestyle & Life questions & Head and neck cancer \\
\hline Anxiety and depression & General everyday life & Social life & Alcohol & Life questions & Swallowing \\
\hline Fear of recurrence & Pain & Relationship with partner & Physical activity & & Speech \\
\hline $\begin{array}{l}\text { Subjective cognitive } \\
\text { functioning }\end{array}$ & Sexuality & Relationship with children & Dietary intake & & Oral function \\
\hline \multirow[t]{9}{*}{ Stress } & Sleep quality & Financial circumstances & Weight & & Neck and shoulder function \\
\hline & Body image & Patient-physician communication & Smoking & & Loss of smell and taste \\
\hline & Fatigue & Return to work & & & $\begin{array}{l}\text { Head and neck cancer specific } \\
\text { lymphedema }\end{array}$ \\
\hline & Diarrhea & & & & Nutritional drink/Tube feeding \\
\hline & Lack of appetite & & & & \\
\hline & Dyspnea & & & & \\
\hline & Nausea or vomiting & & & & \\
\hline & Constipation & & & & \\
\hline & Hearing and tinnitus & & & & \\
\hline
\end{tabular}


feedback in the "Learn" component concludes with a comprehensive self-care advice (tips and tools). All these advices are tailored to the individual cancer survivor (second-degree algorithms).

In the "Act" component, survivors are provided with personalized supportive care options, based on their PRO scores and expressed preferences (e.g., preference for individual therapy versus group therapy) (third-degree algorithms). If a participant has elevated wellbeing risks (orange score), the feedback includes suggestions for self-help interventions. If a participant has "seriously elevated well-being risks" (red score), the feedback includes an advice to contact their own medical specialist or general practitioner.

In appendix 1, a worked example of OncoKompas using a case study is presented. For a clickable demo of the application (in Dutch) or an animation video (in Dutch and English), please visit www.oncokompas.nl.

\section{Outcome measures}

A study-specific survey was composed with items on sociodemographic and clinical factors, a QOL questionnaire (assessed at baseline (T0)), and items on usage and satisfaction (assessed at follow-up (T1)).

\section{Adoption, usage, and satisfaction}

Adoption was defined as the percentage of cancer survivors that agreed to participate in the study and returned the T0 questionnaire and informed consent.

Usage was defined as the percentage of participating cancer survivors that actually used OncoKompas as intended based on the item "Did you fill out and use OncoKompas?" (T1).

General satisfaction was assessed based on the mean score of three study-specific questions: general impression of OncoKompas, the user-friendliness, and the ability to use OncoKompas without assistance (10-point Likert scales: 0 (poor) to 10 (good)). Furthermore, satisfaction was measured with the Net Promoter Score (NPS) with the question "How likely it is that you would recommend OncoKompas to other cancer survivors (10-point Likert scale: 0 (not likely) to 10 (very likely)). The NPS was calculated by dividing the percentage of promoters (who score 9-10) minus the percentage of detractors (who score 0-6). The percentage "passives" (who score 7-8) is not included in calculating NPS. The NPS ranges between -100 and +100 . A positive score is considered good [19].

Additionally, the satisfaction of participants on the three components of OncoKompas was assessed (measure, learn, and act).

\section{Moderating factors}

Sociodemographic (age and gender) and clinical variables (tumor location, tumor stage, type of treatment, and comorbidity) were drafted by a physician from the medical records. Comorbidity was assessed by the use of the Adult Comorbidity Evaluation 27 (ACE-27), a validated chart built instrument, resulting in a total comorbidity score of none, mild, moderate, or severe [20].

The 30-item EORTC QLQ-C30 (version 3.0) includes a global health-related quality of life (HRQOL) scale (two items) and five functional scales: physical functioning, role functioning, emotional functioning, cognitive functioning, and social functioning. There are three symptom scales (nausea and vomiting, fatigue, and pain) and six single items relating to dyspnea, insomnia, loss of appetite, constipation, diarrhea, and financial difficulties. In the present study, the HRQOL scale was used. The scores of the QLQ-C30 are linearly transformed to a scale of $0-100$, with a higher score indicating a higher level of HRQOL [21].

\section{Facilitators and barriers}

After the participants completed the T1 survey, they were interviewed by an oncology nurse to obtain more in-depth insight into barriers and facilitators of the feasibility of OncoKompas.

To evaluate technical issues interfering with the feasibility of OncoKompas, the entries in the helpdesk logbook were evaluated on the type of problem encountered, and if and how the problem was solved.

\section{Data analyses}

Descriptive statistics were used to summarize the adoption, usage, and satisfaction. OncoKompas was defined feasible in case of an adoption and usage grade of more than $50 \%$, a mean satisfaction score of at least 7 , and a positive Net Promoter Score. This definition of feasibility is based on adoption and usage rates reported in previous studies on eHealth applications [22].

Correlations between satisfaction with OncoKompas and gender (male vs. female), age ( $<65$ vs. $>64$ years), comorbidity (none/mild vs. moderate/severe), tumor subsite (oral cavity/oropharynx vs. hypopharynx/larynx vs. other), tumor stage (stage I/II vs. stage III/IV), and treatment modality (surgery alone vs. surgery plus (chemo)radiation vs. (chemo)radiation) were examined using chi-square tests. The outcome variable satisfaction with OncoKompas was not normally distributed and was therefore dichotomised into two categories: a score from 0 to 6 and a score of 7 and higher. The association between satisfaction with OncoKompas and QOL was analyzed using Mann-Whitney U tests. Statistical analyses were 
performed using the Statistical Package for the Social Sciences (IBM SPSS Statistics 20). For all analyses, $p$ values $<0.05$ (two-tailed) were used as criterion for statistical significance.

The structured interview reports by the oncology nurses were analyzed by thematic analyses. Barriers and facilitators towards feasibility of OncoKompas were extracted from the reports and those that were mentioned at least five times are reported. The entries in the logbook with technical difficulties were categorized by type of problem that was encountered. The number of unique problems was counted.

\section{Results}

\section{Adoption and usage}

In total, $106 \mathrm{HNC}$ survivors were asked to participate in the study. The adoption grade was $64 \%$ : 68 out of 106 intended to use OncoKompas and gave informed consent and returned the T0 survey. Reasons for non-consent included a lack of time of the patient and no willingness to travel to the hospital for the nurses' consultation.

In total, 12 participants dropped out during the study $(17.6 \%)$, leaving a study cohort of 56 participants (Table 2). The reasons for dropout included cancer recurrence $(n=2)$, entering palliative care $(n=2)$, family circumstances $(n=1)$, comorbid illness $(n=1)$, tiredness due to which OncoKompas could not be used ( $n=1)$, evaluating the questions in OncoKompas as too confronting $(n=1)$, insufficient Internet skills according to the participant $(n=1)$, and not able to reach participants by telephone $(n=3)$. Of the 56 participants (56 out of 68) who completed the study, 51 survivors filled out OncoKompas completely and as intended (usage grade $91 \%$ ). In total, five of these participants indicated that they received assistance while filling out OncoKompas (with the help of my spouse $(N=4)$ or with the help of my daughter $(N=1))$. Two survivors indicated to have used OncoKompas partially (3.6\%). They mentioned that they only filled out the 'Measure' component in OncoKompas. Three other survivors $(5.4 \%)$ indicated not to have used OncoKompas due to technical reasons; one participant indicated to be hindered by a bug when using the application, while the other two participants indicated that because of a bug in the application they could not continue. Of the participants who encountered technical problems, one survivor contacted the helpdesk. Despite provision of assistance, this participant was not able to complete OncoKompas. Usage grade thus lies between $75 \%$ (51 out of 68 participants (including dropouts)) and $91 \%$ (51 out of 56 participants (excluding dropouts)) who used OncoKompas as intended.
Table 2 Demographic and health characteristics of the participating participants $(N=56)$

\begin{tabular}{|c|c|c|}
\hline & $n$ & $\%$ \\
\hline \multicolumn{3}{|l|}{$\operatorname{Sex}(n, \%)$} \\
\hline Female & 22 & 39.3 \\
\hline Male & 34 & 60.7 \\
\hline \multicolumn{3}{|l|}{ Age in years } \\
\hline Mean (SD) & $59.05(9.85)$ & \\
\hline Minimum & 25 & \\
\hline Maximum & 77 & \\
\hline \multicolumn{3}{|l|}{ Tumor site (n, \%) } \\
\hline Oral cavity and oropharynx & 30 & 53.6 \\
\hline Hypopharynx and larynx & 12 & 21.4 \\
\hline Other & 14 & 25.0 \\
\hline \multicolumn{3}{|l|}{ Comorbidity (ACE-27) $(n, \%)$} \\
\hline None & 16 & 28.6 \\
\hline Mild & 17 & 30.4 \\
\hline Moderate & 18 & 32.1 \\
\hline Severe & 5 & 8.9 \\
\hline \multicolumn{3}{|l|}{ Type of treatment $(n, \%)$} \\
\hline$($ Chemo) radiation therapy $((\mathrm{C}) \mathrm{RT})$ & 27 & 48.2 \\
\hline Surgery & 13 & 23.2 \\
\hline Surgery $+(C) R T$ & 16 & 28.6 \\
\hline \multicolumn{3}{|l|}{ Time since treatment (in months) } \\
\hline Mean (SD) & $12.32(6.5)$ & \\
\hline Minimum & 0 & \\
\hline Maximum & 24 & \\
\hline \multicolumn{3}{|l|}{ Quality of Life (EORTC QLQ C-30) } \\
\hline Mean (SD) & $76.33(16.49)$ & \\
\hline Minimum & 33.33 & \\
\hline Maximum & 100 & \\
\hline
\end{tabular}

\section{Satisfaction}

Most of the participants were satisfied with OncoKompas in general (60.4\%, mean score 6.8, SD 1.2). Participants evaluated OncoKompas as user-friendly (76.0 \%, mean score 7.1, SD 1.6). Participants were able to use OncoKompas without assistance (90.6 \%, mean score 7.8, SD 1.7). The mean satisfaction score was 7.3 (SD 1.5). The Net Promoter Score of OncoKompas was positive 1.9 , consisting of $21 \%$ promoters, $19 \%$ detractors, and $60 \%$ passives.

Regarding the feasibility of the "Measure" component, almost all participants answered all PROs (98\%). For some participants, the PROs were intrusive $(21 \%)$, confusing (29\%), or difficult to answer (37\%). Confusing and difficult questions mentioned by survivors included questions related to God and religion. Questions about sexuality were found intrusive. Almost all participants $(94 \%)$ viewed their wellbeing profile in the "Learn" part of OncoKompas. To most 
participants, the description of their results was clear and understandable (84\%) and easy to find in the application (81\%). More than half of the participants evaluated the information as applicable to themselves (61\%), but less than half evaluated the information of added value for their own health status (43\%). More than half of the participants $(61 \%)$ indicated that the overall picture regarding their results (the compass metaphor) did not add much. Most participants viewed their personalized advices ( $71 \%$ ), and these advices were evaluated as clear $(85 \%)$ and complete (68\%). The amount of supportive care options provided in the "Act" component was considered to be exactly right to most participants $(71 \%)$ or too much $(23 \%)$. More than half of the participants $(57 \%)$ indicated to be interested in one or more of the offered supportive care options and almost a third of these participants subsequently did take action accordingly (29\%). The majority indicated to return to OncoKompas in the future to view their personalized advices and actions once again (71\%).

The helpdesk was contacted for a total of 21 unique problems. The problems mainly consisted of difficulties logging on to the application: due to a browser problem $(n=2)$, loss of password $(n=2)$, expiration of security certificate of the application $(n=3)$, use of a tablet $(n=2)$, blockage by firewall $(n=2)$, no compliance of the computer with application requirements $(n=1)$, and other reasons $(n=5)$. Furthermore, an error message appeared $(n=2)$ and the button to print the results was not visible $(n=2)$.

\section{Factors associated with satisfaction with OncoKompas}

Satisfaction with OncoKompas was not significantly associated with age $\left(X^{2}(1, \mathrm{~N}=53)=0.26, p=0.61\right)$, gender $\left(X^{2}(1\right.$, $N=53)=0.58, p=0.45)$, tumor location $\left(X^{2}(2\right.$, $N=53)=5.49, p=0.06)$, tumor stage $\left(X^{2}(1\right.$, $N=53)=0.00, p=0.97)$, type of treatment $\left(X^{2}(2\right.$,
$N=53)=3.38, p=0.19)$, comorbidity $\left(X^{2}(1\right.$, $N=53)=0.034, p=0.85)$, and HRQOL $(p=0.35)$.

\section{Barriers and facilitators}

In total, seven barriers towards the feasibility of OncoKompas were mentioned at least five times (Table 3): (1) The application did not fully take into account other diseases that participants suffered from, (2) the amount of information in the application was too much, (3) items regarding existential issues were difficult to answer and too much oriented towards religion, (4) participants did not find the results completely applicable to their personal situation (they experienced their symptoms in a different way), (5) participants found a lapsed time of 2 years since treatment to introduce the application too long (these participants often already found a solution to the experienced problems or learned to live with them), (6) the description of participant's overall well-being was suboptimal, either considered confronting or meaningless, and (7) participants found some items in the application confusing making it difficult to answer them truthfully.

Six facilitators were mentioned at least five times (Table 3): (1) the user-friendliness of the application, (2) it's informative nature, (3) the provision of a clear overview to the participant of their personal situation and options, (4) the clarity of the items in the questionnaire, (5) the usefulness of the application in general, and (6) the particular usefulness of the application for participants who are very ill or experience many symptoms.

\section{Discussion}

The aim of this study was to investigate the feasibility of the eHealth application OncoKompas aiming to facilitate and innovate cancer survivorship care. Our results show that OncoKompas is feasible with an adoption grade of $64 \%$, a

Table 3 Barriers and facilitators (mentioned at least five times)

\begin{tabular}{|c|c|c|c|}
\hline Barriers & $\begin{array}{l}\text { No. of times } \\
\text { mentioned }\end{array}$ & Facilitators & $\begin{array}{l}\text { No. of times } \\
\text { mentioned }\end{array}$ \\
\hline $\begin{array}{l}\text { The application did not fully take into account other } \\
\text { diseases that participants suffered from }\end{array}$ & 11 & The user-friendliness of the application & 13 \\
\hline The amount of information in the application was too much & 9 & The informative nature of the applications & 7 \\
\hline $\begin{array}{l}\text { Items regarding existential issues were difficult to answer } \\
\text { and too much oriented towards religion }\end{array}$ & 9 & $\begin{array}{l}\text { The provision of a clear overview to the participant of their } \\
\text { personal situation and options }\end{array}$ & 6 \\
\hline $\begin{array}{l}\text { Participants did not find the results completely applicable } \\
\text { to their personal situation. }\end{array}$ & 8 & The clarity of the items in the questionnaire. & 6 \\
\hline $\begin{array}{l}\text { Participants found a lapsed time of } 2 \text { years since treatment } \\
\text { to introduce the application too long }\end{array}$ & 7 & The usefulness of the application in general & 5 \\
\hline $\begin{array}{l}\text { The description of participant's overall well-being was } \\
\text { suboptimal }\end{array}$ & 6 & $\begin{array}{l}\text { The particular usefulness of the application for participants } \\
\text { who are very ill or experience many symptoms }\end{array}$ & 5 \\
\hline $\begin{array}{l}\text { Participants found some items in the application confusing } \\
\text { making it difficult to answer them truthfully. }\end{array}$ & 6 & & \\
\hline
\end{tabular}


usage grade between 75 and $91 \%$, a mean satisfaction score of 7.3, and a positive Net Promoter Score. Almost all participants were able to use OncoKompas. The few participants that were not able to use OncoKompas seemed to be hindered by insufficient eHealth skills. These findings are in line with previous studies that examined the feasibility of eHealth applications in clinical practice showing that eHealth applications are acceptable to many participants but not to all [23-26].

Our study showed no associations with sociodemographic factors. Also in previous studies, no significant associations between use of eHealth and gender were found [25, 27, 28]. Previous studies revealed mixed results concerning the association between age and use of eHealth applications [25-29]. Increased age seems to be associated with less use of the Internet, although this association has become less strong in recent years, probably due to the availability and increased familiarity of Internet [26]. A review by Or and Karsh revealed that most studies did not show significant relationships between eHealth acceptance and age [25]. Our study results match these findings.

In our study, there were no significant associations between satisfaction with OncoKompas and clinical factors or QOL. Previous studies that focused on health and treatment factors showed that participants who use eHealth are healthier than participants who do not use eHealth $[30,31]$. A better health status seems to lead to a better acceptance of Internet applications [32]. However, in other studies, the opposite was found [33, 34].

Other factors than sociodemographic and clinical factors and QOL may be of more interest in investigating why participants use (or not use) eHealth applications. Adoption rate of eHealth can be predicted by the way an eHealth application is rated in terms of usefulness and ease of use, and the self-efficacy of participants regarding information technology [25, 35]. In the present study, only participants with sufficient (self-reported) computer skills were included. Facilitators associated with the feasibility of OncoKompas included the user-friendliness of the application and its informative nature. Ease of use was also determined in previous studies as an important factor for the acceptance of eHealth applications [25]. Important barriers included the feeling that the results did not completely reflect the personal situation, the large amount of information in the application, and difficulties answering some of the items. The barrier regarding the time investment required to complete the application has been reported in previous studies as well. Length and information overload have been found to be important reasons to quit using an online application [36-38]. Individualized feedback has been found to be related to sustained intervention use and less dropout [39, 40].

The strength of our study is that we used mixed methods, providing in-depth insight into the feasibility of eHealth applications in clinical practice.

In the present study, only participants were included with access to the Internet at home. Therefore, we do not have good insight into the representativeness of our study sample. The positive attitude of participants towards OncoKompas might not be generalizable to all HNC survivors. Another limitation concerns the small sample size, which may have hampered testing the associations. Finally, in this study, participants had access to the eHealth application for only 2 weeks. Further research is needed on the feasibility in the longer term.

\section{Conclusions}

OncoKompas is considered feasible, but our results also show that improvements can be made to enhance the feasibility and increase the satisfaction among cancer survivors. The PROs can be further investigated and possibly be reduced. It is also important to look at the phrasing of individual items, because of the barrier that answering some of the items was difficult to participants. However, in order to ensure accuracy of the individualized feedback, we have to balance the time it takes to use OncoKompas, measurement precision, and tailoring towards a personalized tool. It is clear that any eHealth application will not be suitable for all participants, due to different needs, preferences, and coping styles of cancer survivors. It is worthwhile to obtain more insight into how further tailoring of eHealth applications and more sophisticated marketing strategies can be applied leading to applications that are attractive to more participants and hereby increase adoption and usage.

\section{Compliance with ethical standards}

Conflict of interest The authors declare that they have no competing interests.

Appendix 1: The flow of OncoKompas To provide more insight into how OncoKompas works, the flow of OncoKompas is presented to illustrate the successive steps a user takes in OncoKompas that lead him or her to personalized supportive care options. We will use a case study to make the OncoKompas components comprehensible and provide insight into the way the responses of users to the PROs lead to personalized advice. This case study concerns a 49-year old female head and neck cancer survivor named Mary. She has finished treatment for laryngeal cancer 6 months prior to using OncoKompas. She is married and has two children.

Login When entering OncoKompas for the first time, a user is requested to create an account to login. Mary is asked to provide her personalia, date of birth, and e-mail address.

Measure After the login phase of OncoKompas is completed, a user enters the "Measure" component. In this component, users are presented with PROs targeting the following quality of life domains; physical functioning, psychological functioning, social functioning, lifestyle, and existential issues, completed with a tumor-specific domain containing specific PROs for head and neck cancer survivors. Users are asked to complete all PROs. The PRO system consists of dynamic questionnaires. This means that the system automatically determines whether sequence 
questions are appropriate. In our case study, Mary's answers to the screening questions regarding sexual functioning (i.e. 'Are you willing to answer questions regarding your sexuality?", "Have you been sexually active?", "Have you experienced problems while being sexually active?") and mood result in the provision of elaborated questionnaires (Female Sexual Function Index (FSFI) ${ }^{1}$ and the Hospital Anxiety and Depression Scale (HADS) $)^{2}$ on these topics. In her answers to the FSFI, Mary indicates that she experienced almost no sexual desire in the past 4 weeks and is dissatisfied with her sexual relationship and emotional bond during sexual intercourse with her husband. On the HADS, she indicates that she finds it difficult to enjoy the things she used to enjoy, does not feel cheerful, and has trouble to laugh and see the funny side of things.

Learn When the PROs are completed, the data from the "Measure" component are processed in real-time and linked to tailored feedback to the user in the "Learn" component. All algorithm calculations are based on available cut-off scores or are defined based on Dutch practice guidelines, literature searches, and/or consensus by teams of experts. Feedback is provided to the user on the levels of the addressed topics in the PROs (e.g., sexual problems), by means of a three-color system: green (no elevated well-being risks), orange (elevated well-being risks), and red (seriously elevated well-being risks) (first-degree algorithms).

In our case study, Mary receives an "orange" score on sexual functioning, since her score on the FSFI was less than 26.55 (cut-off score for an orange profile $)^{3}$. She receives a "red" score regarding her mood, because her score on the depression subscale of the HADS is 10 (cut-off score for a red profile $\geq 8)^{4}$. When Mary wants to read elaborated personalized information about her well-being risks on a specific topic, e.g., sexual functioning, she can do so by mouse clicking the topic. This will enable her to read more information about how sexual problems can arise after cancer treatment, and how these may influence her quality of life.

The elaborated information is followed by specific attention for clusters of interrelated symptoms. In Mary's case, she suffers from depressive symptoms, which could be related to her sexual problems. Therefore, the user is provided with feedback on the possible interrelation between his or her symptoms. For Mary, this information consists of how her depressive symptoms may influence her libido or may negatively impact the way she experiences sex. She also receives information about how her depressive symptoms may be influenced by her sexual problems, e.g., frustration to accept changes regarding sexuality due to cancer (treatment), relationship problems, or a feeling of inferiority caused by her sexual problems.

The feedback in the "Learn" component concludes with comprehensive self-care advice (tips and tools). All these advices are tailored to the individual user (second-degree algorithms). In our case study, Mary is provided with a brochure about sexuality and cancer from the Dutch Cancer Society, combined with the advice to select a supportive care option from the "Act" component to address her sexual problems.

Act After finishing the "Learn" component, users are automatically directed to the "Act" component. In this component of OncoKompas, users are provided with personalized supportive care options, based on their PRO scores and expressed preferences (e.g., preference for individual therapy versus group therapy) (third-degree algorithms). If a participant has elevated well-being risks (orange score), the feedback includes suggestions for (guided) self-help interventions. If a participant has "seriously elevated well-being risks" (red score), the feedback includes an advice to contact their own medical specialist or general practitioner. In Mary's case, she is provided with three supportive care options regarding her sexual functioning: the possibility of online sex therapy (e.g., via mental health care providers that offer internet therapy), the website of the Rutgers Nisso group (www.seksualiteit.nl) that provides tips and online self-help exercises to cope with problems regarding sexual functioning and has a specific information section regarding sexual functioning after cancer treatment, and the website of the Dutch Society of Sexology (www.nvvs.info) that provides elaborated information regarding problems with sexual functioning and what the role of a sexual therapist entails. Also, three alternative supportive care options are provided: the advice to contact her treating physician, her general practitioner, or a sexual therapist. Regarding her depressive symptoms, she is also advised to contact her physician or her general practitioner and is able to mouse click a link to a website that has a list of psychologists specialized in oncology to make a well informed choice. If a user wishes to do so, he or she is able to print their OncoKompas results and bring these with them to their physicians consultation.

1 Rosen R, Brown C, Heiman J, Leiblum S, Meston C, Shabsigh R et al., Ferguson D, D'Agostino R Jr. The Female Sexual Function Index (FSFI): a multidimensional self-report instrument for the assessment of female sexual function. J Sex Marital Ther 2000; 26(2):191-208.

2 Zigmond AS, Snaith RP: The hospital anxiety and depression scale. Acta Psychiatr Scand 1983, 67: 361-370.

3 Wiegel M, Meston C, Rosen R., The female sexual function index (FSFI): cross-validation and development of clinical cutoff scores.

4. Bjelland, I., Dahl, A. A., Haug, T. T., \& Neckelmann, D. (2002). The validity of the Hospital Anxiety and Depression Scale: An updated literature review. Journal of Psychosomatic Research, 52(2), 69-77.

Open Access This article is distributed under the terms of the Creative Commons Attribution-NonCommercial 4.0 International License (http:// creativecommons.org/licenses/by-nc/4.0/), which permits any noncommercial use, distribution, and reproduction in any medium, provided you give appropriate credit to the original author(s) and the source, provide a link to the Creative Commons license, and indicate if changes were made.

\section{References}

1. A. de Graeff, J. R. de Leeuw, W. J. Ros, G. J. Hordijk, G. H. Blijham, and J. A. Winnubst, "Long-term quality of life of patients with head and neck cancer.," Laryngoscope, vol. 110, no. 1, pp. 98-106, Jan. 2000.

2. I. M. Verdonck-de Leeuw, S. E. Eerenstein, M. H. Van der Linden, D. J. Kuik, R. de Bree, and C. R. Leemans, "Distress in spouses and patients after treatment for head and neck cancer.," Laryngoscope, vol. 117, no. 2, pp. 238-241, Feb. 2007.

3. N. K. Aaronson, V. Mattioli, O. Minton, J. Weis, C. Johansen, S. O. Dalton, I. M. Verdonck-de Leeuw, K. D. Stein, C. M. Alfano, A. Mehnert, A. de Boer, and L. V. van de Poll-Franse, "Beyond treatmentpsychosocial and behavioural issues in cancer survivorship research and practice,” Eur J Cancer Suppl, vol. 12, no. 1, pp. 54-64, Jun. 2014.

4. E. C. Moser and F. Meunier, "Cancer survivorship: a positive sideeffect of more successful cancer treatment," Eur J Cancer Suppl, vol. 12, no. 1, pp. 1-4, Jun. 2014.

5. F. Jansen, C. F. van Uden-Kraan, V. van Zwieten, B. I. Witte, and I. M. Verdonck-de Leeuw, "Cancer survivors' perceived need for supportive care and their attitude towards self-management and eHealth.," Support Care Cancer, vol. 23, no. 6, pp. 1679-1688, Jun. 2015.

6. R. McCorkle, E. Ercolano, M. Lazenby, D. Schulman-Green, L. S. Schilling, K. Lorig, and E. H. Wagner, "Self-management: enabling and empowering patients living with cancer as a chronic illness.," CA Cancer J Clin, vol. 61, no. 1, pp. 50-62, Jan. 2011.

7. A. J. Mitchell, "Short screening tools for cancer-related distress: a review and diagnostic validity meta-analysis.," J Natl Compr Cancer Netw, vol. 8, no. 4, pp. 487-494, Apr. 2010.

8. A. J. Mitchell, "Screening for cancer-related distress: when is implementation successful and when is it unsuccessful?," Acta Oncol, vol. 52, no. 2, pp. 216-224, Feb. 2013. 
9. M. I. Fitch, "Supportive care framework.," Can Oncol Nurs J, vol. 18, no. 1, pp. 6-24, Jan. 2008.

10. J. E. W. C. van Gemert-Pijnen, N. Nijland, M. van Limburg, H. C. Ossebaard, S. M. Kelders, G. Eysenbach, and E. R. Seydel, "A holistic framework to improve the uptake and impact of eHealth technologies.," J. Med. Internet Res., vol. 13, no. 4, p. e111, Jan. 2011.

11. J. N. Stinson, G. C. Petroz, B. J. Stevens, B. M. Feldman, D. Streiner, P. J. McGrath, and N. Gill, "Working out the kinks: testing the feasibility of an electronic pain diary for adolescents with arthritis.," Pain Res. Manag., vol. 13, no. 5, pp. 375-382, Jan..

12. R. van der Vaart, C. H. C. Drossaert, E. Taal, and M. A. F. J. van de Laar, "Patient preferences for a hospital-based rheumatology interactive health communication application and factors associated with these preferences.," Rheumatology $($ Oxford)., vol. 50, no. 9, pp. 1618-1626, Sep. 2011.

13. S. Lubberding, C. F. van Uden-Kraan, E. A. Te Velde, P. Cuijpers, C. R. Leemans, and I. M. Verdonck-de Leeuw, "Improving access to supportive cancer care through an eHealth application: a qualitative needs assessment among cancer survivors.," J. Clin. Nurs., Feb. 2015.

14. R. de Bree, I. M. Verdonck-de Leeuw, A. L. Keizer, A. Houffelaar, and C. R. Leemans, "Touch screen computer-assisted health-related quality of life and distress data collection in head and neck cancer patients.," Clin Otolaryngol, vol. 33, no. 2, pp. 138-142, Apr. 2008.

15. S. Vosbergen, E. K. Laan, E. B. Colkesen, M. A. J. Niessen, R. A. Kraaijenhagen, M.-L. Essink-Bot, and N. Peek, "Evaluation of enduser satisfaction among employees participating in a web-based health risk assessment with tailored feedback.," J. Med. Internet Res., vol. 14, no. 5, p. e140, Jan. 2012.

16. S. M. Kelders, W. T. M. Pots, M. J. Oskam, E. T. Bohlmeijer, and J. E. W. C. van Gemert-Pijnen, "Development of a web-based intervention for the indicated prevention of depression.," BMC Med Inform Decis Mak, vol. 13, p. 26, Jan. 2013.

17. R. G. Booth, "Examining the functionality of the DeLone and McLean information system success model as a framework for synthesis in nursing information and communication technology research.," Comput. Inform. Nurs., vol. 30, no. 6, pp. 330-345, Jun. 2012.

18. S. Lubberding, C.F. Van Uden-Kraan, W.J.M.J. Cuijpers, C.R. Leemans, and I.M. Verdonck-de Leeuw, "Developing an eHealth portal targeting survivorship cancer care: a user-centred design approach.," Psycho-Oncology, vol. 22, pp. 15-16, 2013.

19. Reichheld F, Markey R (2011) the ultimate question 2.0 (revised and expanded edition): how net promoter companies thrive in a customer-driven world. Harvard Business School Press:224

20. J. F. Piccirillo, R. M. Tierney, I. Costas, L. Grove, and E. L. Spitznagel, "Prognostic importance of comorbidity in a hospital-based cancer registry.," Jama, vol. 291, no. 20, pp. 2441-2447, May 2004.

21. N. K. Aaronson, S. Ahmedzai, B. Bergman, M. Bullinger, A. Cull, N. J. Duez, A. Filiberti, H. Flechtner, S. B. Fleishman, and J. C. de Haes, "The European organization for research and treatment of cancer QLQC30: a quality-of-life instrument for use in international clinical trials in oncology.," J Natl Cancer Inst, vol. 85, no. 5, pp. 365-376, Mar. 1993.

22. I. C. Cnossen, C. F. van Uden-Kraan, R. N. P. M. Rinkel, I. J. Aalders, C. J. T. de Goede, R. de Bree, P. Doornaert, D. H. F. Rietveld, J. A. Langendijk, B. I. Witte, C. R. Leemans, and I. M. Verdonck-de Leeuw, "Multimodal guided self-help exercise program to prevent speech, swallowing, and shoulder problems among head and neck cancer patients: a feasibility study.," J. Med. Internet Res., vol. 16, no. 3, p. e74, Jan. 2014.

23. C. M. Ruland, T. Andersen, A. Jeneson, S. Moore, G. H. Grimsbø, E. Børøsund, and M. C. Ellison, "Effects of an internet support system to assist cancer patients in reducing symptom distress: a randomized controlled trial.," Cancer Nurs, vol. 36, no. 1, pp. 6-17, Jan. 2013.

24. C. M. Ruland, R. M. Maffei, E. Børøsund, A. Krahn, T. Andersen, and G. H. Grimsbø, "Evaluation of different features of an eHealth application for personalized illness management support: cancer patients' use and appraisal of usefulness.," Int J Med Inform, vol. 82, no. 7, pp. 593-603, Jul. 2013.

25. C. K. L. Or and B.-T. Karsh, 2009 "A systematic review of patient acceptance of consumer health information technology.," J Am Med Inform Assoc, vol. 16, no. 4, pp. 550-560, Jan..

26. Y. K. Bartlett, D. L. Selby, A. Newsham, A. Keding, D. Forman, J. Brown, G. Velikova, and P. Wright, "Developing a useful, user-friendly website for cancer patient follow-up: users' perspectives on ease of access and usefulness.," Eur J Cancer Care (Engl)., vol. 21, no. 6, pp. 747-757, Nov. 2012.

27. E. Børøsund, M. Cvancarova, M. Ekstedt, S. M. Moore, and C. M. Ruland, "How user characteristics affect use patterns in web-based illness management support for patients with breast and prostate cancer.," J. Med. Internet Res., vol. 15, no. 3, p. e34, Jan. 2013.

28. A.-M. Wiggers, N. Peek, R. Kraaijenhagen, and M. Jaspers, "Determinants of eligibility and use of ehealth for cardiac rehabilitation patients: preliminary results.," Stud Health Technol Inform, vol. 205, pp. 818-822, Jan. 2014.

29. C. F. Van Uden-Kraan, C. H. C. Drossaert, E. Taal, W. M. Smit, H. J. Bernelot Moens, and M. A. F. J. Van de Laar, "Determinants of engagement in face-to-face and online patient support groups.," $J$. Med. Internet Res., vol. 13, no. 4, p. e106, Jan. 2011.

30. S. M. Kelders, J. E. W. C. Van Gemert-Pijnen, A. Werkman, N. Nijland, and E. R. Seydel, "Effectiveness of a Web-based intervention aimed at healthy dietary and physical activity behavior: a randomized controlled trial about users and usage.," J. Med. Internet Res., vol. 13, no. 2, p. e32, Jan. 2011.

31. M. W. Verheijden, M. P. Jans, V. H. Hildebrandt, and M. HopmanRock, "Rates and determinants of repeated participation in a webbased behavior change program for healthy body weight and healthy lifestyle.," J. Med. Internet Res., vol. 9, no. 1, p. e1, Jan. 2007.

32. Y. M. Chae, H. J. Park, J. G. Cho, G. D. Hong, and K. A. Cheon, "The reliability and acceptability of telemedicine for patients with schizophrenia in Korea.," J Telemed Telecare, vol. 6, no. 2, pp. 8390, Jan. 2000

33. J.-G. Jeannot, F. Froehlich, V. Wietlisbach, B. Burnand, O. Terraz, and J.-P. Vader, "Patient use of the internet for health care information in Switzerland.," Swiss Med Wkly, vol. 134, no. 21-22, pp. 307-312, May 2004.

34. Millard RW, Fintak PA (2002) Use of the internet by patients with chronic illness. Dis Manag Heal Outcomes 10(3):187-194

35. Venkatesh FD, Morris V, Davis MG (2003) User acceptance of information technology: toward a unified view. MIS Quarterly. 27:425-478

36. V. Voncken-Brewster, H. Tange, A. Moser, Z. Nagykaldi, H. de Vries, and T. van der Weijden, "Integrating a tailored e-health self-management application for chronic obstructive pulmonary disease patients into primary care: a pilot study.," BMC Fam Pract, vol. 15, p. 4, Jan. 2014.

37. W. Brouwer, A. Oenema, R. Crutzen, J. de Nooijer, N. K. de Vries, and J. Brug, "What makes people decide to visit and use an internetdelivered behavior-change intervention?," Health Educ, vol. 109, no. 6, pp. 460-473, Oct. 2009.

38. J. O. Prochaska, "Multiple health behavior research represents the future of preventive medicine.," Prev Med (Baltim)., vol. 46, no. 3, pp. 281-285, Mar. 2008.

39. J. R. Schubart, H. L. Stuckey, A. Ganeshamoorthy, and C. N. Sciamanna, "Chronic health conditions and internet behavioral interventions: a review of factors to enhance user engagement.," Comput Inform Nurs, vol. 29, no. 2 Suppl, pp. TC9-T20, Feb. 2011.

40. W. Brouwer, W. Kroeze, R. Crutzen, J. de Nooijer, N. K. de Vries, J. Brug, and A. Oenema, "Which intervention characteristics are related to more exposure to internet-delivered healthy lifestyle promotion interventions? A systematic review.," J. Med. Internet Res., vol. 13, no. 1, p. e2, Jan. 2011. 